\title{
Defining the pathogenic involvement of desmoglein 4 in pemphigus and staphylococcal scalded skin syndrome
}

\author{
Takeshi Nagasaka, ${ }^{1}$ Koji Nishifuji, ${ }^{1}$ Takayuki Ota, ${ }^{1}$ Neil V. Whittock, ${ }^{2}$ and Masayuki Amagai ${ }^{1}$ \\ ${ }^{1}$ Department of Dermatology, Keio University School of Medicine, Shinjuku-ku, Tokyo, Japan. \\ ${ }^{2}$ Institute of Biochemical and Clinical Science, Peninsula Medical School, Exeter, United Kingdom.
}

\begin{abstract}
Desmogleins (Dsgs), cadherin-type cell adhesion molecules, are targeted in skin-blistering diseases such as pemphigus and staphylococcal scalded skin syndrome (SSSS). The role of Dsg4, a new isoform, was investigated in these diseases. Dsg 4 was recognized by 30 (77\%) of 39 pemphigus sera containing anti-Dsg1 IgG but not by 16 pemphigus sera containing no anti-Dsg1 IgG or by 34 normal control sera. The Dsg4 immunoreactivity of these sera was abolished by removal of anti-Dsg1 IgG. Conversely, the removal of anti-Dsg4 IgG from pemphigus sera reduced the immunoreactivity against Dsg1 only $13.8 \% \pm 8.8 \%(n=23)$ and did not affect its ability to induce blisters in neonatal mice. IgG that was affinity-purified on Dsg4 recognized Dsg1 but failed to induce blisters, while IgG purified on Dsg1 from the same pemphigus foliaceus sera induced blisters. Thus, pemphigus sera show Dsg 4 reactivity due to cross-reactivity of a subset of anti-Dsg1 IgG, and the Dsg4/Dsg1-cross-reacting IgG has no demonstrable pathogenic effect. In addition, Dsg 4 was not cleaved by exfoliative toxins that induce blisters in SSSS. These findings suggest that Dsg4 may play a role other than adhesion and that the cross-reactivity of desmoglein autoantibodies should be factored into the framework of future studies of autoimmune mechanisms in pemphigus.
\end{abstract}

\section{Introduction}

Desmoglein (Dsg), a major transmembrane component in desmosomes, plays a critical role not only in the cell-cell adhesion of epithelial cells but also in the morphogenesis of epithelial tissues $(1,2)$. Since the identification of the desmoglein cDNAs in the early 1990s, 3 isoforms of desmoglein, i.e., Dsg1, Dsg2, and Dsg3, have been described (3-6). Dsg2 is expressed in all desmosomepossessing tissues, which include simple epithelia and the myocardium, while the expression of Dsg1 and Dsg3 is restricted to stratified squamous epithelium (6). Recently, a fourth member of the desmoglein family, Dsg4, was identified based on an analysis of the genomic structure of the desmosomal cadherin gene cluster on human chromosome 18q12 $(7,8)$.

The desmogleins are targeted in inherited and acquired skin diseases as well as in skin infections. Mutations in the DSG1 gene cause striate palmoplantar keratoderma (MIM 148700), which is an autosomal dominant skin disease that is characterized by linear and focal hyperkeratosis of the palms and soles, probably owing to haploinsufficiency of Dsg1 (9). Dsg1 and Dsg3 are autoimmune targets in pemphigus, which is characterized by blisters and erosions of the skin and mucous membranes $(5,10)$. The classic forms of pemphigus are divided into 3 subtypes according to anti-desmoglein $\mathrm{Ab}$ profile (11, 12): patients with pemphigus foliaceus (PF) have only antiDsg1 IgG autoantibodies; patients with the mucosal dominant type of pemphigus vulgaris (PV) have only anti-Dsg3 IgG

Nonstandard abbreviations used: Dsg, desmoglein; ET, exfoliative toxin; hDsg4, human Dsg4; mDsg4, mouse Dsg4; PF, pemphigus foliaceus; PV, pemphigus vulgaris; SSSS, staphylococcal scalded skin syndrome.

Conflict of interest: The authors have declared that no conflict of interest exists.

Citation for this article: J. Clin. Invest. 114:1484-1492 (2004).

doi:10.1172/JCI200420480. autoantibodies; and patients with the mucocutaneous type of PV have both anti-Dsg3 and anti-Dsg1 IgG autoantibodies. The disruption of desmoglein-dependent cell-cell adhesion by IgG autoantibodies represents the basic pathophysiology underlying blister formation in pemphigus, and the sites of blister formation in the skin and mucous membranes are explained by "the desmoglein compensation theory" $(13,14)$.

In addition to its involvement in these inherited and acquired blistering skin diseases, Dsg1 is also targeted by the exfoliative toxins (ETs) of Staphylococcus aureus, leading to bullous impetigo, which is a common bacterial infection of children, as well as staphylococcal scalded skin syndrome (SSSS), which is the generalized form found in newborns, young children, and adults with renal failure and/or immunocompromised status $(15,16)$. Among the 4 known isoforms of ET, ETA, ETB, and ETD bind and cleave the extracellular domain of Dsg1 once after glutamic acid residue 381 between the EC3 and EC4 domains in a specific manner (16-19). Thus, these ETs act as serine proteases with strict specificity for Dsg1, and they induce superficial blisters in the epidermis that are clinically and histologically similar to those seen in PF.

Mutations in the newly identified DSG4 gene cause impaired hair follicle development, which results in inherited hypotrichosis in humans $(7,20,21)$ and abnormal hair with bulbous degenerative changes in the lanceolate hair mouse and rat $(7,22)$. It has been claimed also that Dsg 4 acts as an autoantigen in PV, based on IB analysis showing that $2 \mathrm{PV}$ sera recognized a bacterial recombinant protein that represented an extracellular domain of Dsg4 (7). It is puzzling, however, that the phenotypes of the inherited form and the acquired form are quite different; the phenotype caused by the mutated DSG4 is rather restricted to the hair and hair follicles, without apparent blister formation in the interfollicular skin or mucous membranes, as is found in patients with PV (23). 


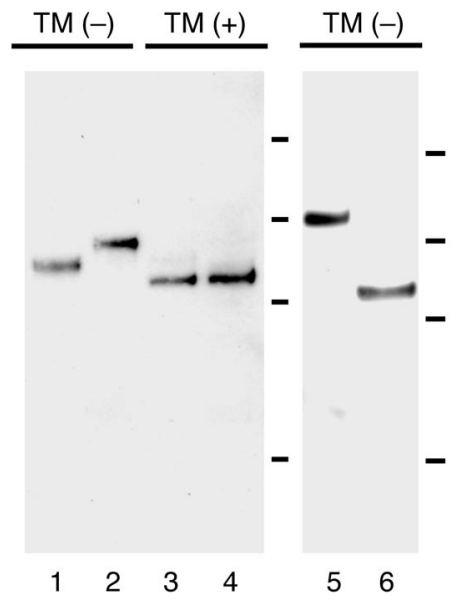

In this study, we investigated the involvement of Dsg4, which is expressed in the superficial epidermis (7), in the skin-blistering diseases pemphigus and SSSS. We examined the reactivity against Dsg4 of sera derived from patients with different types of pemphigus and the specificity of anti-Dsg4 IgG

\section{Figure 1}

Production of the fusion construct of the secreted form of the entire extracellular domain of hDsg4 with the E-tag and His-tag (hDsg4-His) using baculovirus expression (lane 1; 91.7-kDa protein) and by mammalian expression in $\mathrm{CHO}$ cells (lane 2; 98.6-kDa protein). The molecular weights of nonglycosylated forms of hDsg4-His produced in the presence of tunicamycin (TM) by baculovirus (lane 3 ) and in $\mathrm{CHO}$ cells (lane 4) were identical (87.6 kDa). The entire extracellular domain of hDsg4 fused with constant region of human IgG1 and His-tag (hDsg4IgHis; lane 5; $119.3 \mathrm{kDa}$ ) and $\mathrm{mDsg} 4$ fused with the E-tag and Histag (mDsg4-His; lane 6; $86.8 \mathrm{kDa}$ ) were also produced by baculovirus expression. The bars indicate the molecular weight standards (from top to bottom: $150,100,75$, and $50 \mathrm{kDa}$ ).

autoantibodies. We compared anti-Dsg4 autoantibodies to other known pemphigus autoantibodies and investigated their role in the pathogenesis of blister formation. We also determined whether Dsg4 is a target of the ETs that cause superficial epidermal blisters in SSSS.

It is important to understand the involvement of Dsg4 in pemphigus and SSSS, not only to elucidate the functional role it may play in vivo but also to build a framework for future investigation into the autoimmune mechanisms of pemphigus.
A
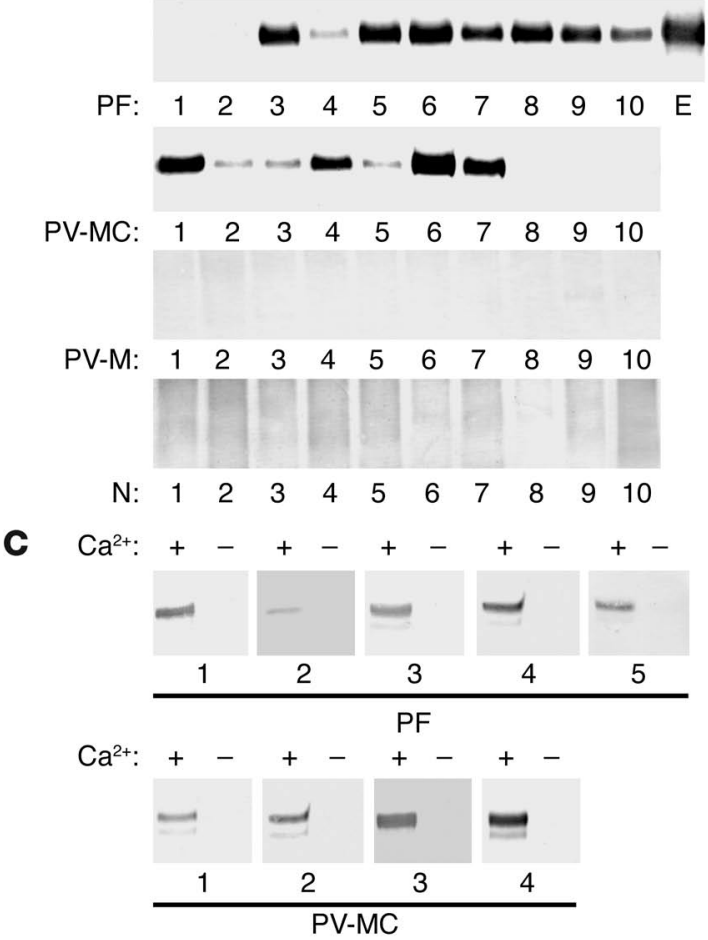

B

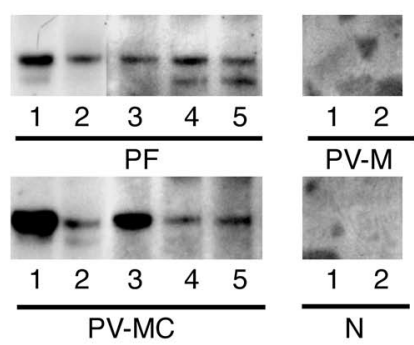

D

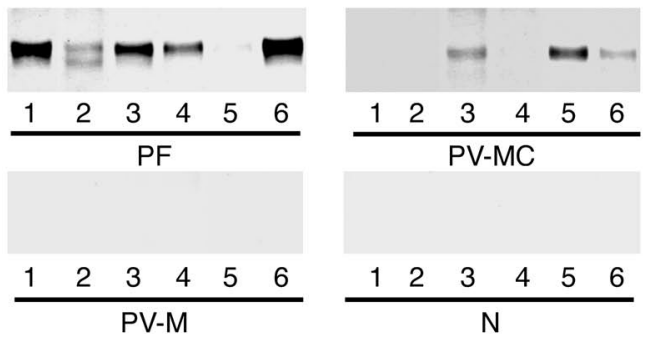

Figure 2

IP-IB analysis of recombinant Dsg4 proteins in pemphigus and normal sera. (A) The hDsg4-His, which was produced by baculovirus expression, was immunoprecipitated with sera from patients with PF, mucocutaneous PV (PV-MC), mucosal dominant PV (PV-M), or normal individuals $(\mathrm{N})$. The results from 10 representative sera are shown. The anti-E-tag mAb (E) was used as the positive control. Most of the PF and PV-MC sera, but none of the PV-M or N sera, reacted with hDsg4-His. (B) hDsg4-His, which was produced by eukaryotic expression in CHO cells, was immunoprecipitated by PF and PV-MC sera, but not by PV-M or normal sera. (C) The calcium dependency of the Dsg4 reactivity was determined. Removal of calcium by dialysis abolished the reactivities of the PF and PV-MC sera against Dsg4, which indicates that these sera recognize calcium-dependent conformational epitopes on Dsg4. (D) The mDsg4-His produced by baculovirus expression was immunoprecipitated by PF, PV-MC, PV-M, and N sera. Similar to the findings with hDsg4, many of the PF and PV-MC sera, but none of the $\mathrm{PV}-\mathrm{M}$ or $\mathrm{N}$ sera, reacted with the mDsg4. 
A

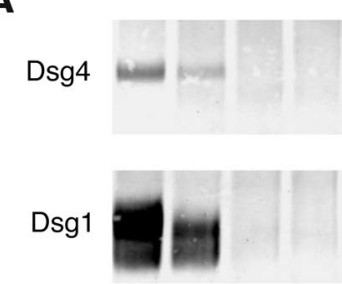

Preadsorption with Dsg1

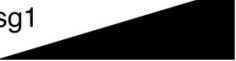

C

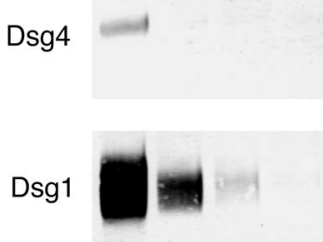

Dsg3

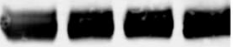

Preadsorption with Dsg1
B

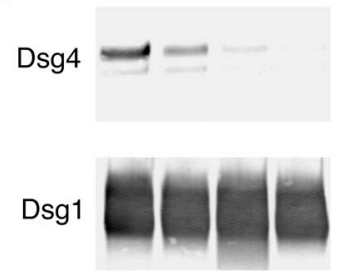

Preadsorption with Dsg4

D

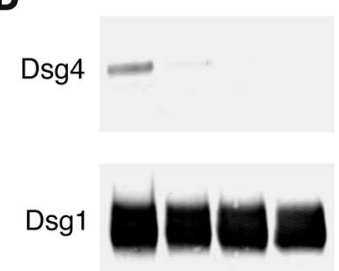

Dsg3

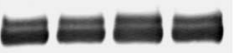

Preadsorption with Dsg4

\section{Figure 3}

Elimination of Dsg4 reactivity by removal of anti-Dsg1 IgG autoantibodies. Anti-Dsg1 IgG autoantibodies were preadsorbed from PF sera $(\mathbf{A})$ or PV-MC sera (C) using various amounts of hDsg1-IgHis. When the anti-Dsg1 IgG autoantibodies were removed, Dsg4 reactivity was eliminated, while Dsg3 reactivity was unaltered. Similarly, the anti-Dsg4 IgG autoantibodies were preadsorbed from PF sera (B) or PV-MC sera (D) using various amounts of hDsg4-IgHis. Although the anti-Dsg4 IgG autoantibodies were removed, Dsg1 reactivity was not reduced significantly. The heights of the black triangles indicate the amounts of hDsg1-IgHis ( $\mathbf{A}$ and $\mathbf{C})$ or hDsg4-IgHis ( $\mathbf{B}$ and $\mathbf{D})$ that were used for preadsorption.

supernatant via dialysis (Figure 2C). None of the $20 \mathrm{PF}$ and 0 of 16 mucocutaneous PV sera reacted with the denatured hDsg4-His as examined by IB analysis (data not shown).

In addition, we expressed hDsg4-His in CHO cells as a 98.6-kDa protein (Figure 1, lane 2). The difference of the molecular weights between that expressed by baculovirus and that expressed in $\mathrm{CHO}$ cells was a result of glycosylation because the molecular weights for nonglycosylated forms produced in the presence of tunicamycin were identical (Figure 1, lanes 3 and 4$)$. The PF sera $(n=6)$ and mucocutaneous PV sera $(n=5)$ that reacted with the baculovirusexpressed hDsg4-His also reacted with the hDsg4-His produced by the $\mathrm{CHO}$ cells (Figure 2B). Neither the mucosal dominant PV sera $(n=2)$ nor the normal sera $(n=2)$ showed any reactivity against the $\mathrm{CHO}$ cell-expressed hDsg4-His. This finding excludes the possibility that the reactivities against hDsg4-His are owing to the generation of neo-epitopes during baculovirus expression.

These data indicate that a subset of pemphigus sera react with calcium-dependent conformational epitopes on Dsg4. Interestingly, both the PF sera and mucocutaneous PV sera contained anti-Dsg1 IgG autoantibodies, while the mucosal dominant PV sera contained no anti-Dsg1 IgG, but did contain anti-Dsg3 IgG autoantibodies. These disparate reactivity patterns raised the possibility that the reactivities against Dsg4 might be the result of cross-reactivities of anti-Dsg1 IgG autoantibodies.

Reactivity of pemphigus sera against Dsg4 is eliminated by removal of the anti-Dsg1 IgG autoantibodies. To determine whether Dsg4 reactivity involved anti-Dsg1 IgG autoantibodies, we preincubated the sera that showed reactivity against Dsg4 with various amounts of hDsg1-IgHis (25) and performed IP-IB assays against hDsg1-His, hDsg3-His, and hDsg4-His. When the PF sera that reacted with Dsg4 were examined, the reactivity against hDsg4-His was eliminated in a dose-dependent fashion by preincubation with hDsg1-IgHis (Figure 3A). Dsg4 reactivity

Table 1

Immunoreactivity of pemphigus sera against Dsg4

\begin{tabular}{|c|c|c|c|c|c|c|}
\hline \multirow[t]{2}{*}{ Subtypes } & \multirow[t]{2}{*}{$n$} & \multicolumn{2}{|c|}{ Reactivity against } & \multirow[t]{2}{*}{ Dsg4 reactivity ${ }^{A}$} & \multirow{2}{*}{$\begin{array}{l}\text { Elimination of Dsg4 } \\
\text { reactivity by Dsg1 }\end{array}$} & \multirow{2}{*}{$\begin{array}{l}\text { Reduction of Dsg1 reactivity } \\
\text { by Dsg4 immunoadsorption }\end{array}$} \\
\hline & & Dsg1 & Dsg3 & & & \\
\hline PF & 20 & + & - & $17(85.0 \%)$ & $17(100 \%)$ & $10.7 \% \pm 5.8 \%(n=11)$ \\
\hline PV-MCD & 19 & + & + & $13(68.4 \%)$ & $13(100 \%)$ & $17.1 \% \pm 10.5 \%(n=12)$ \\
\hline PV-ME & 16 & - & + & $0(0 \%)$ & NA & $N A$ \\
\hline Normal & 34 & - & - & $0(0 \%)$ & NA & NA \\
\hline
\end{tabular}

AThe reactivity against Dsg4 was examined by a combined IP-IB analysis using hDsg4-His as the substrate. BThe elimination of Dsg4 reactivity was examined by removing anti-Dsg1 IgG autoantibodies with hDsg1-IgHis. CThe reduction rate of Dsg1 reactivity was examined by competition ELISA against Dsg1 using hDsg4-His as a competitor. DMucocutaneous PV. EMucosal dominant PV. 


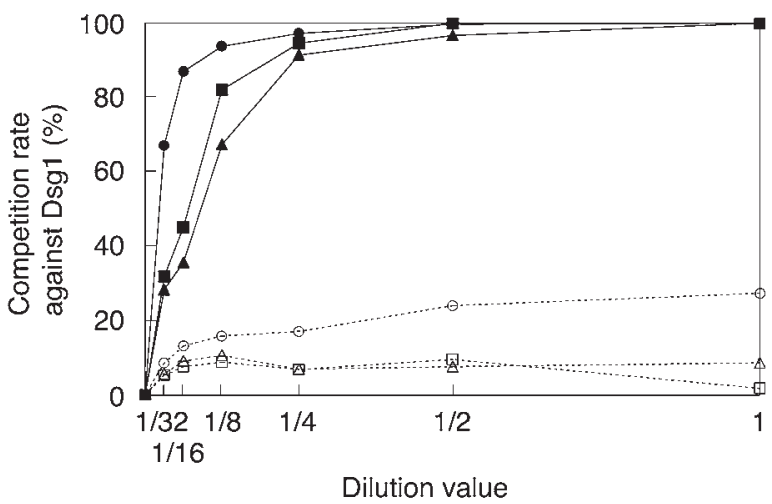

Figure 4

Competition ELISA against Dsg1 with Dsg4 or Dsg1 as a competitor. Three representative PF sera were incubated with serial dilution of concentrated culture supernatants containing hDsg4-His (open symbols) or hDsg1-His (closed symbols) and subjected to Dsg1 ELISA. The competition rate by Dsg4 increased in a dose-dependent fashion, although its plateau level was no more than $25 \%$.

was abolished completely when anti-Dsg1 IgG was removed. The removal of Dsg4 reactivity by preincubation with hDsg1-IgHis was observed for 17 of $17(100 \%)$ of the Dsg4-reacting PF sera (Table 1). When the mucocutaneous PV sera that reacted with Dsg4 were examined, the reactivity against hDsg4-His was also eliminated in a dose-dependent fashion by preincubation with hDsg1-IgHis (Figure 3C). The removal of the anti-Dsg1 IgG autoantibodies by hDsg1-IgHis completely abolished the reactivity against $\mathrm{hDsg} 4-\mathrm{His}$, whereas it did not affect the reactivity against hDsg3-His. The removal of Dsg4 reactivity with hDsg1IgHis was observed for 13 of $13(100 \%)$ of the Dsg4-reacting mucocutaneous PV sera (Table 1).

We also removed the IgG autoantibodies that reacted with Dsg4 and then examined their reactivities against Dsg1 or Dsg3. In the case of the PF sera, the removal of Dsg4 reactivity by preincubation with hDsg4-IgHis (Figure 1, lane 5) did not significantly affect the reactivity against hDsg1-His $(n=5)$ (Figure $3 \mathrm{~B}$ ). Similarly, in the case of the mucocutaneous PV sera, the removal of Dsg4 reactivity did not change the reactivity against $\mathrm{hDsg} 1$-His or $\mathrm{hDsg} 3$-His $(n=6)$ (Figure 3D). To further quantitate the IgG fraction cross-reacting with Dsg4 in anti-Dsg1 IgG autoantibodies, we performed competition ELISA against Dsg1 using various concentrations of hDsg4-His as competitors. Competition increased in a dosedependent fashion and reached a plateau at higher concentration (representative data are shown in Figure 4). The observed plateau level of competition for each serum should represent the IgG fraction cross-reacting with Dsg4 in anti-Dsg1 IgG autoantibodies. We tested 11 PF sera and 12 mucocutaneous PV sera that showed the reactivity with Dsg4 (Table 1). The Dsg1 reactivity was reduced $10.7 \% \pm 5.8 \%$ in PF sera and $17.1 \% \pm 10.5 \%$ in mucocutaneous PV sera by removing anti-Dsg4 IgG. Thus, on average, $13.8 \% \pm 8.8 \%$ of the anti-Dsg1 IgG autoantibodies were considered to show the cross-reactivity with $\operatorname{Dsg} 4(n=23)$.

Furthermore, we used the hDsg4-His column to affinity-purify the IgG autoantibodies that reacted with Dsg4 from the PF sera and determined the reactivities of these sera against Dsg1 and Dsg3 (Figure 5). The affinity-purified anti-Dsg4 IgG autoantibodies reacted with hDsg1-His but not with hDsg3-His $(n=2)$.
Taken together, these findings indicate that there were essentially no IgG autoantibodies that reacted exclusively with Dsg4 in pemphigus and that the Dsg4 reactivity found in the PF sera and mucocutaneous PV sera is owing to the cross-reactivity of a subset of anti-Dsg1 IgG autoantibodies.

The Dsg4/Dsg1-cross-reacting IgG autoantibodies do not play a significant pathogenic role in blister formation in pemphigus. To evaluate the pathogenic relevance of Dsg4/Dsg1-cross-reacting IgG autoantibodies in the pemphigus sera, we performed passive transfer assays using neonatal mice (26). To ensure that the IgG autoantibodies that reacted with hDsg4 also reacted with mouse Dsg4 (mDsg4), we conducted IP-IB assays using mDsg4-His (Figure 1, lane 6; Figure 2D). Similar to the findings with hDsg4, 11 of $12 \mathrm{PF}$ sera and 3 of 6 mucocutaneous PV sera reacted with mDsg4-His, while 0 of 6 mucosal dominant PV sera and 0 of 12 normal control sera recognized it. In addition, Dsg4 is indeed expressed in neonatal mouse epidermis. The expression of $D s g 4$ was demonstrated by RT-PCR in the various sites of the skin (ventral and dorsal skin, tail, and footpad) as well as in the tongue, but not in simple epithelia such as liver (Figure 6). In the same condition, the expression of $D s g 1 \alpha, D s g 1 \beta, D s g 1 \gamma$, and $D s g 3$ was demonstrated in the stratified squamous epithelia but not in liver, except for $D s g 1 \gamma$, as previously described $(5,27,28)$. Because essentially all IgG that reacts with Dsg 4 cross-reacts with Dsg1 in pemphigus sera, the following experiments do not reflect sole effects by Dsg4 inhibition, but rather combined effects by Dsg1 and Dsg4 inhibition.

We removed the IgG autoantibodies that reacted with Dsg4 by immunoadsorption with hDsg4-His and prepared IgG from the treated PF sera, which no longer reacted with Dsg4 (Figure 5). To compare the pathogenic strength of the PF sera with and without Dsg4 immunoadsorption in a quantitative fashion, the IgG fractions were serially diluted and injected into neonatal mice (Table 2). Mice injected with PF no. 1 serum IgG after the removal of anti-Dsg4 IgG developed extensive blisters 10 hours after injection (Figure 7B). The histology showed loss of cell adhesion in the upper part of the epidermis, a typical finding in PF (Figure 7F). Direct immunofluorescence of the mouse skin showed human IgG deposition on keratinocyte cell surfaces (Figure 7J). Mice injected with PF no. 1 serum IgG without hDsg4-His immunoadsorption
A

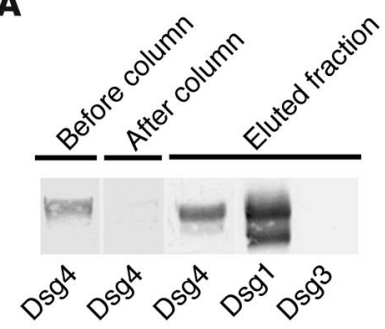

B

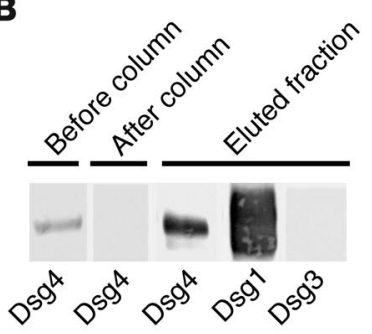

\section{Figure 5}

Immunoadsorption and purification of Dsg4-reactive IgG autoantibodies from PF sera. Two of the PF sera (A and B) were applied to a column that contained $\mathrm{hDsg} 4-\mathrm{His}$, and the Dsg4 reactivities of the sera were examined by IP-IB analysis both before and after column purification. We injected the IgG fractions, which were prepared from the sera with or without immunoadsorption by $\mathrm{hDsg} 4-\mathrm{His}$, into neonatal mice to examine their pathogenic activities (see Figure 7). IgG that was affinity-purified on the hDsg4-His column was eluted (eluted fraction) and examined for its reactivities against Dsg1, Dsg3, and Dsg4. The IgG affinity-purified on Dsg4 cross-reacted with Dsg1 but not with Dsg3. 


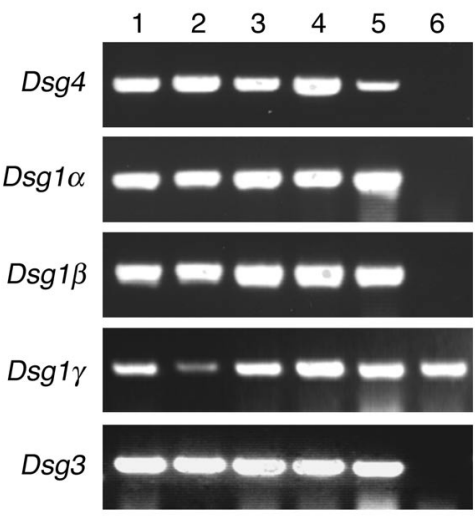

Figure 6

Dsg4 expression in neonatal mouse epidermis. Total RNA was extracted from various sites of the skin (lane 1, ventral skin; lane 2, dorsal skin; lane 3, footpad; lane 4, tail) and tongue (lane 5) and liver (lane 6) in neonatal mice and then subjected to RT-PCR for detection of expression for mouse $D s g 4, D s g 1 \alpha, D s g 1 \beta, D s g 1 \gamma$, and $D s g 3$. Note that $D s g 4$ is expressed in various sites of mouse stratified squamous epithelia, including footpad and tongue, where no hair follicles are present, but not in the liver.

developed blisters at the same time (10 hours) after the injection (Figure 7A), and their histological and immunopathological features were essentially identical (Figure 7, E and I). Mice injected with 2-fold dilutions of the IgG fractions developed blisters after 13 hours. Those injected with IgG diluted 4-fold or more developed no blisters, regardless of the removal of anti-Dsg4 IgG (Table 2). In contrast, mice injected with IgG after the removal of anti-Dsg1 showed no gross or histological signs of blister formation (Table 2). Similarly, the titration of PF no. 2 serum IgG showed essentially no difference in pathogenic strength, with or without immunoadsorption with hDsg4-His (Table 2).

Serial dilutions of IgG that was affinity-purified in parallel on hDsg4-His or hDsg1-His from the same volume of PF sera were injected into neonatal mice to directly compare their pathogenic strengths (Table 3). Mice injected with Dsg4/Dsg1-cross-reacting IgG (Figure 5) from PF no. 1 serum did not develop blisters grossly or histologically (Figure 7, C and G), despite positive in vivo IgG deposition in the skin (Figure $7 \mathrm{~K}$ ); those injected with affinitypurified anti-Dsg1 IgG developed extensive superficial blisters (Figure 7, D, H, and L). Twofold diluted, but not 4-fold diluted, anti-Dsg1 IgG was sufficient to induce blistering, while diluted anti-Dsg4/Dsg1-cross-reacting IgG was not (Table 3). Similarly, affinity-purified Dsg4/Dsg1-cross-reacting IgG from PF no. 2 serum also failed to induce blisters, while the affinity-purified anti-Dsg1 IgG did induce blisters, even after 2-fold dilution (Table 3). The titers of circulating IgG were determined by indirect immunofluorescence using cryo-sectioned neonatal mouse skin, and they were 2-fold to 4-fold higher in mice injected with the original concentration of anti-Dsg1 IgG (1:320 to $1: 640)$ than those injected with the Dsg4/Dsg1-cross-reacting IgG (1:160 to $1: 320)$ for both PF no. 1 and PF no. 2 sera. The difference in these titers were considered to be reflecting the amounts of the respective IgG included in PF sera (Table 1, Figure 4).
These findings indicate that the Dsg4/Dsg1-cross-reacting IgG fraction found in PF sera does not play a major pathogenic role in blister formation in PF.

Dsg4 is not digested by the exfoliative toxins of S. aureus. The ETs produced by $S$. aureus cause bullous impetigo, which is a common bacterial infection of children, as well as SSSS, which is its generalized form. Three isoforms of ET (ETA, ETB, and ETD) are known to digest Dsg1. To determine whether Dsg4 is cleaved directly by these ETs, we incubated hDsg4-His and mDsg4-His with ETA, $\mathrm{ETB}$, and ETD in vitro at $37^{\circ} \mathrm{C}$ for 1 hour (Figure 8). None of the ETs cleaved hDsg4-His, while all of the ETs cleaved hDsg1His. Similarly, none of the ETs cleaved mDsg4-His, while they all digested $\mathrm{mDsg} 1 \alpha$-His. These findings indicate that Dsg 4 is not targeted by ETs. Therefore, Dsg4 does not appear to be involved in the pathogenesis of blister formation in impetigo or SSSS.

\section{Discussion}

Desmosomes are essential adhesion structures in most types of epithelium. Their transmembrane components are desmosomal cadherins, desmogleins, and desmocollins (1). Until recently, 3 human and mouse desmogleins had been characterized, namely, Dsg1, Dsg2, and Dsg3, which are expressed in tissue- and differentiation-specific manners. Recent detailed genomic analyses identified a fourth member of the desmoglein family, Dsg4, in humans and mice $(7,8)$. It has been elegantly demonstrated that mutations in the DSG4 gene cause inherited localized hypotrichosis in humans and the lanceolate hair phenotype in mice (7). In this study, we investigated whether Dsg4 is targeted in the skin autoimmune disease of pemphigus, as well as in the skin infection disease of impetigo and SSSS.

Because the IgG autoantibodies in pemphigus recognize conformational epitopes (29-31), we produced the secreted form of the extracellular domain of Dsg 4 by baculovirus expression and examined the reactivities of pemphigus sera by IP-IB analysis. We found that 17 of 20 (85\%) PF sera and 13 of 19 (68\%) mucocutaneous PV sera, both of which contained anti-Dsg1 IgG autoantibodies, reacted with Dsg4, but none of 16 mucosal dominant PV sera and none of 34 normal control sera did (Figure 2, Table 1). Interestingly, Dsg4 reactivity was eliminated in all of the pemphigus sera that showed Dsg4 reactivity by the removal of anti-Dsg1 IgG by preadsorption with hDsg1-IgHis (Figure 3, Table 1). IgG that was affinity-purified on Dsg4 immunoprecipitated not only Dsg4,

Table 2

Titration of pathogenic activity of PF IgG with removal of anti-Dsg4 or antiDsg1 IgG autoantibodies in passive transfer with neonatal mice

\begin{tabular}{ccccc} 
Serum used & $\begin{array}{c}\text { Amount of } \\
\text { IgG injected }\end{array}$ & \multicolumn{3}{c}{ Mice with gross blisters } \\
No removal & $\begin{array}{c}\text { Removal of } \\
\text { anti-Dsg4 IgG }\end{array}$ & $\begin{array}{c}\text { Removal of } \\
\text { anti-Dsg1 IgG }\end{array}$ \\
PF no. 1 & Original & $2 / 2(10 \mathrm{~h})^{A}$ & $2 / 2(10 \mathrm{~h})$ & $0 / 2$ \\
& 2 Diluted & $2 / 2(13 \mathrm{~h})$ & $2 / 2(13 \mathrm{~h})$ & $0 / 2$ \\
& 4 Diluted & $0 / 2$ & $0 / 2$ & $0 / 2$ \\
PF no. 2 & 8 Diluted & $0 / 2$ & $0 / 2$ & $0 / 2$ \\
& Original & $2 / 2(11 \mathrm{~h})$ & $2 / 2(11 \mathrm{~h})$ & $\mathrm{ND}$ \\
& 2 Diluted & $2 / 2(12 \mathrm{~h})$ & $2 / 2(12 \mathrm{~h})$ & $\mathrm{ND}$ \\
& 4 Diluted & $0 / 2$ & $0 / 2$ & $\mathrm{ND}$ \\
& 8 Diluted & $0 / 2$ & $0 / 2$ & $\mathrm{ND}$
\end{tabular}

ATime required for development of gross blisters. 

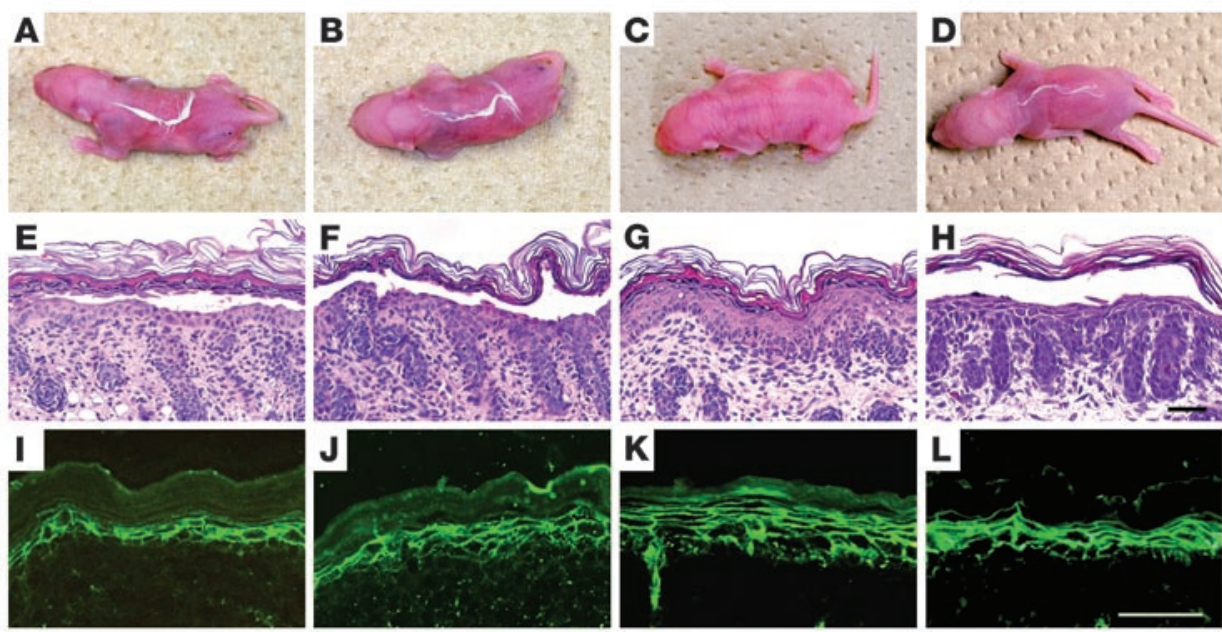

$\mathbf{H}$

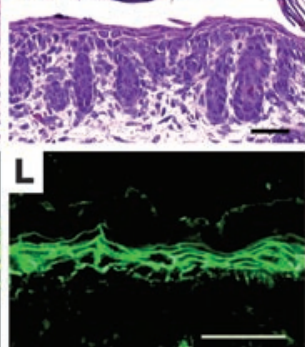

\section{Figure 7}

Lack of pathogenic involvement of Dsg4/Dsg1-cross-reacting IgG in blister formation in pemphigus. Pathogenic activity of each IgG fraction was determined by passive transfer into neonatal mice. Mice were injected with: IgG prepared from $P F$ no. 1 serum without $(\mathbf{A}, \mathbf{E}$, and $\mathbf{I})$ or with $(\mathbf{B}, \mathbf{F}$, and $\mathbf{J})$ removal of anti-Dsg4 IgG, affinity-purified Dsg4/Dsg1-cross-reacting IgG (C, G, and K), or anti-Dsg1 IgG (D, H, and $\mathbf{L})$. Gross appearance of mice (A-D), histology $(\mathbf{E}-\mathbf{H})$, and direct immunofluorescence of the skin $(\mathbf{I}-\mathbf{L})$ are shown for each treatment. The induction of blisters in neonatal mice was equivalent whether anti-Dsg4 IgG was removed (B, F, and $\mathbf{J}$ ) or not removed (A, E, and I) from the IgG fraction. Affinitypurified Dsg4/Dsg1-cross-reacting IgG failed to induce blisters, despite positive in vivo IgG deposition on keratinocyte cell surfaces (C, G, and $\mathbf{K})$; anti-Dsg1 IgG affinity-purified from the same volume of PF sera induced extensive blisters (D, H, and $\mathbf{L})$. Scale bars: $100 \mu \mathrm{m}$.

but also Dsg1 (Figure 5). Conversely, the removal of anti-Dsg4 IgG from the PF sera did not significantly reduce the reactivity against Dsg1, but decreased it only $13.8 \% \pm 8.8 \%$ (Figures 3 and 4 ; Table 1). These findings indicate that although many of the $\mathrm{PF}$ and mucocutaneous PV sera react with Dsg4, the Dsg4 reactivity is a result of the cross-reactivity of a small subset of antiDsg1 autoantibodies (Figure 9). There were essentially no IgG autoantibodies that reacted exclusively with Dsg4 in pemphigus, and all fractions of anti-Dsg4 IgG found in pemphigus sera cross-reacted with both Dsg1 and Dsg4. The 2 PV sera used in the previous study were obtained from patients with the mucocutaneous PV phenotype and should contain both anti-Dsg1 and anti-Dsg3 IgG autoantibodies (7); their reactivities against Dsg4 may also have been owing to the cross-reactivity of anti-Dsg1 IgG autoantibodies. Furthermore, the immunoadsorption of antiDsg4 IgG from the PF sera did not block the induction of blisters, while the removal of anti-Dsg1 IgG blocked blister formation in the passive transfer model using neonatal mice (Figure 7, Table 2). Affinity-purified Dsg4/Dsg1-cross-reacting IgG failed to induce blisters, and anti-Dsg1IgG affinity-purified in parallel from the same volume of PF sera induced blisters even when diluted 2-fold (Figure 7, Table 3). These results, however, do not necessarily indicate that the Dsg4/Dsg1-cross-reacting IgG plays no pathogenic role at all because it could inhibit the Dsg1 function to a certain degree. Therefore, anti-Dsg1 IgG autoantibodies alone are necessary and sufficient for blister formation in PF, and the Dsg4/ Dsg1-cross-reacting IgG autoantibodies play only a minor pathogenic role in blister formation in pemphigus.

When the amino acid sequences of the desmoglein isoforms were compared, the overall protein identity of hDsg4 was higher with Dsg3 (50\%) than with Dsg1 (41\%) (8). Comparisons of the N-ter-
Dsg4. Conversely, it is also speculated that immunoreactivity against foreign antigens of virus or bacteria may react against epitopes common to Dsg1 and Dsg4 by molecular mimicry, and subsequent epitope spreading may occur on Dsg1. Thus, the presence of Dsg1/Dsg4-cross-reactive IgG autoantibodies provides an important framework for future studies of autoimmune mechanisms in pemphigus (Figure 9).

ETs produced by $S$. aureus cause bullous impetigo and SSSS by cleaving the extracellular domain of Dsg1 once, after glutamic acid residue $381(16,19)$. None of the 3 known isoforms of ET, i.e., ETA, ETB, or ETD, cleaved the extracellular domains of the human and mouse Dsg4 proteins (Figure 8, A and B). This result appears to be consistent with the alignment of the human Dsg1, Dsg3, and Dsg4 protein sequences immediately before the cleavage site, which indicates that Dsg4 is less conserved with Dsg1 (only 3 of 12 identical residues) than with Dsg3 (9 of 12 identical residues)

\section{Table 3}

Pathogenic activity of affinity-purified IgG autoantibodies on Dsg4 and Dsg1 in passive transfer with neonatal mice

$\begin{array}{cccc}\text { Serum used } & \begin{array}{c}\text { Amount of } \\ \text { IgG injected }\end{array} & \begin{array}{c}\text { Mice with gross blisters } \\ \text { Purified Dsg4/Dsg1 } \\ \text { cross-reacting IgG }\end{array} & \begin{array}{c}\text { Purified } \\ \text { anti-Dsg1 IgG }\end{array} \\ \text { PF no. 1 } & \text { Original } & 0 / 5 & 2 / 2(11 \mathrm{~h})^{\mathrm{A}} \\ & \text { 2 diluted } & 0 / 2 & 1 / 1(12 \mathrm{~h}) \\ & \text { 4 diluted } & 0 / 2 & 0 / 2 \\ \text { PF no. } 2 & \text { Original } & 0 / 2 & 2 / 2(9 \mathrm{~h}, 10 \mathrm{~h}) \\ & \text { 2 diluted } & 0 / 2 & 2 / 2(10 \mathrm{~h}, 20 \mathrm{~h}) \\ & \text { 4 diluted } & 0 / 2 & 0 / 2\end{array}$

ATime required for development of gross blisters. 


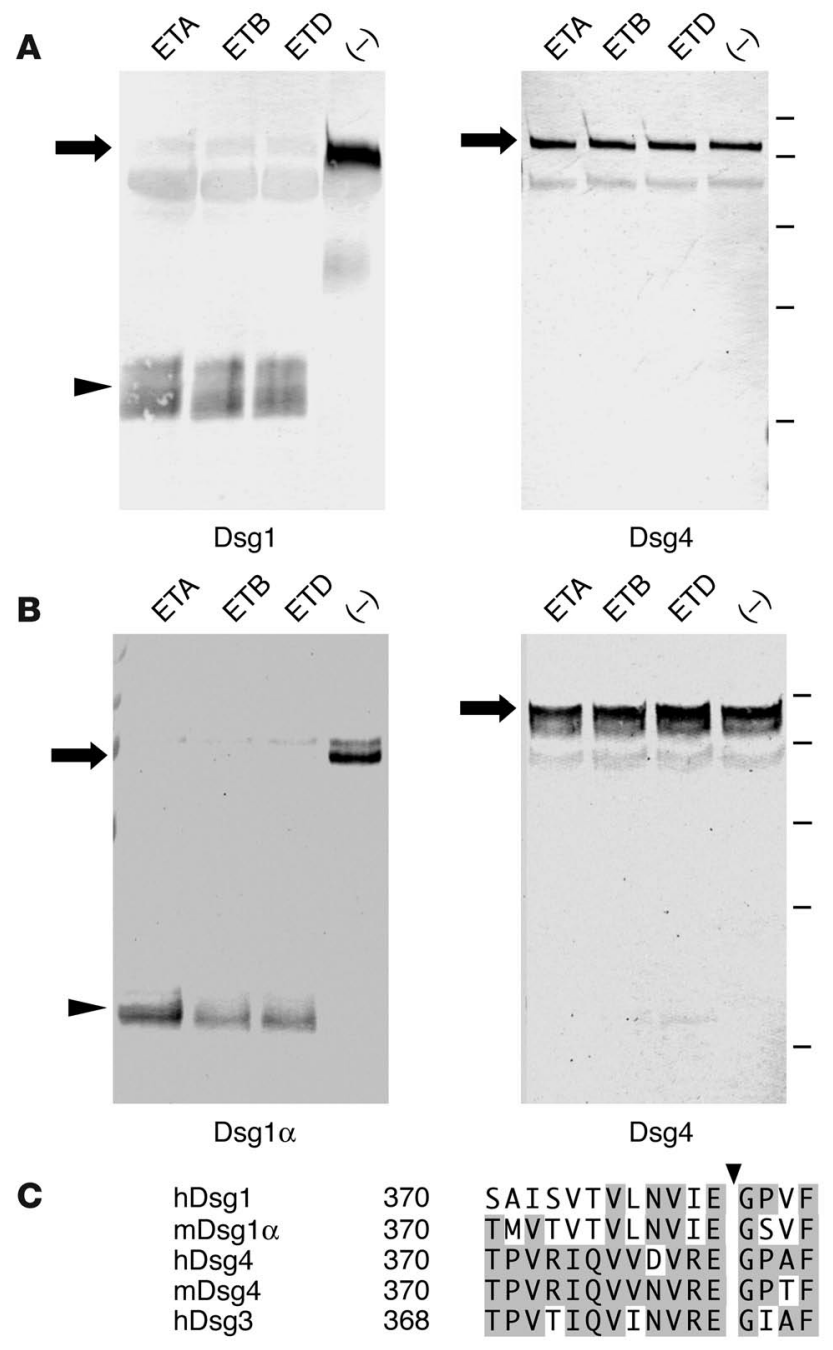

(Figure 8C). Dsg4 is expressed in the suprabasal epidermis, as determined by immunostaining with chicken Abs that were raised against a specific peptide of Dsg4 (7). Dsg2 is not digested by ETA (Nishifuji and Amagai, unpublished data) but is expressed exclusively in the basal cell layer at a low level (35). Therefore, in the epidermis of patients with bullous impetigo and SSSS, ETs digest Dsg1, while leaving intact Dsg4 behind in the upper layers of the human epidermis. This raises the question as to why Dsg4 cannot compensate for the impaired adhesive function of Dsg1 in the human epidermis. Patients with mutations in the DSG4 gene show hypotrichosis, but do not manifest blisters or erosions in the skin (7). In addition, mice that lack Dsg4 expression show abnormal hair development, but they do not have blisters or erosions in the interfollicular skin (23). These findings suggest that Dsg4 may not play a significant role in the cell-cell adhesion of keratinocytes in the interfollicular epidermis and that Dsg4 may fulfill a role other than one in cell-cell adhesion in the epidermis.

Hair follicles are complex organs of the skin and contain compositionally different desmosomes in their different compartments $(36,37)$. In mice, Dsg3 plays an important role in the anchorage of telogen hair, as the inhibition of Dsg3, either by genetic abrasion or IgG autoantibodies, leads to telogen-specific hair loss (38-40). Dsg 4 seems to be the principal desmosomal cadherin in the anagen

\section{Figure 8}

Lack of in vitro digestion of Dsg4 by ETs. Human (A) and mouse (B) Dsg1 $(\alpha)$-His and Dsg4-His were incubated with ETA, ETB, ETD, or PBS alone (-) in vitro at $37^{\circ} \mathrm{C}$ for 1 hour. In both the human and mouse cases, Dsg1 $(\alpha)$ was cleaved by all of the ETs, while Dsg4-His was not digested by any of the ETs. The arrows and arrowhead indicate intact recombinant Dsg proteins and the cleaved protein, respectively. The bars indicate the molecular weight standards (from top to bottom: 100, $75,50,37$, and $25 \mathrm{kDa}$ ). (C) Amino acid sequence alignment of human Dsg1, mouse Dsg1 $\alpha$, human Dsg4, mouse Dsg4, and human Dsg3 around the cleavage site of ETs (arrowhead). Amino acid residues that are conserved in human or mouse Dsg4 are shaded.

hair follicle, and genetic disruption of Dsg4 leads to a transient, intermittent defect in hair follicle development and keratinization, with resultant periodic nodules along the hair shaft and increased hair fragility (7). It will be intriguing to determine whether Dsg4 is targeted in the development of acquired alopecia such as alopecia areata, which is postulated to be an organ-specific autoimmune disease, although the target antigen has not been identified. Future studies that explore the involvement of Dsg4 in various skin diseases represent an exciting field in medicine.

\section{Methods}

Human sera. Sera were obtained from 20 patients with PF, 16 patients with the mucosal dominant type of $\mathrm{PV}$, and 19 patients with the mucocutaneous type of PV, each of whom had his or her diagnosis confirmed by clinical, histological, and immunopathological findings. All of the PF sera were positive for anti-Dsg1 IgG and negative for anti-Dsg3 IgG, as determined by ELISA $(33,41)$. Two of the PF sera that were obtained by plasmapheresis were used for the passive transfer study using neonatal mice (26). All of the mucosal dominant-type PV sera used in this study were positive for antiDsg3 IgG and negative for anti-Dsg1 IgG. All of the mucocutaneous-type PV sera used in this study were positive for both anti-Dsg3 and anti-Dsg1 IgG. The sera from 34 normal individuals were used as controls.

Production of Dsg 4 recombinant protein by baculovirus and mammalian expression. The cDNA that encodes the entire extracellular domain of hDsg4, including the signal peptide and prosequence, was obtained by RT-PCR using total RNA extracted from normal human scalp skin and the following specific primers for hDsg4 (GenBank accession number, AY177664): forward primer, 5'-AAGCCATGGATTGGCTCTTCTTCAGA-3', and reverse primer, 5'-TGGCTCGAGACCAACATTTGAAACTCC-3'. The amplified

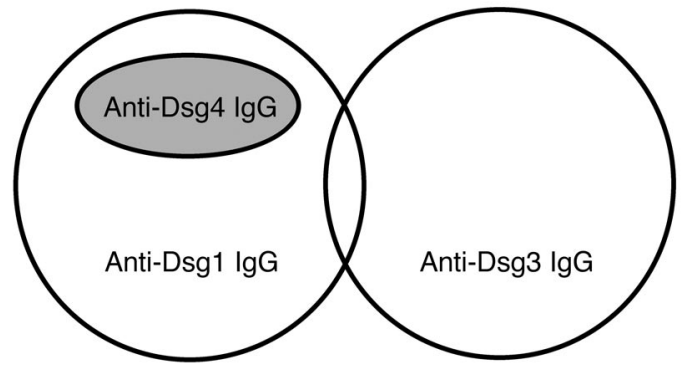

Figure 9

Anti-Dsg IgG autoantibodies in pemphigus. Two kinds of IgG autoantibodies have been characterized in pemphigus, anti-Dsg1 and anti-Dsg3 IgG. Cross-reactivity between Dsg1 and Dsg3 is only occasionally found in pemphigus sera. The identification of the Dsg4 isoform and the characterization of its involvement in pemphigus have revealed that anti-Dsg4 IgG is a cross-reacting subset of anti-Dsg1 lgG. 
cDNA was digested with NcoI and XhoI and ligated into the pQE-TriSystem vector (QIAGEN Inc.) to generate $\mathrm{pQE}$-hDsg4-His (42). The E-tag and His-tag were inserted in tandem at the $\mathrm{C}$-terminal end of this protein. The cDNA that encodes the entire extracellular domain of $\mathrm{mDsg} 4$ was also obtained by RT-PCR of total RNA from ICR mouse skin with the following specific primers for mDsg4 (GenBank accession number, NM181564): forward primer, 5'-CGAAACCATGGACTGGCTTCTCTTCAGAAA-3', and reverse primer, 5'-TGGCTCGAGTCCGACGTTTGACTGCCTAACTCCAT- $3^{\prime}$. The cDNA was digested with $\mathrm{NcoI}$ and $\mathrm{XhoI}$ and ligated into the pQE-TriSystem, thereby generating PQE-mDsg4-His. In addition, a fusion between the entire extracellular domain of hDsg4 and the constant region of human IgG1 and the His-tag (hDsg4-IgHis) was constructed in the baculovirus transfer vector pEVmod (25). Nucleotide sequencing of the hDsg 4 constructs revealed 2 nucleotides that are different from the published sequence (G285A and C495T, nucleotide numbering from the ATG translation initiation codon; GenBank, AF177664). These nucleotides do not cause any amino acid changes. The mDsg 4 construct did not have any nucleotide changes (GenBank, NM181564).

To produce the recombinant human and mouse Dsg4 proteins (hDsg4-His, mDsg4-His, and hDsg4-IgHis), the pQE-hDsg4-His, pQE-mDsg4-His, and pEVmod-hDsg4IgHis plasmids were cotransfected with Sapphire Baculovirus DNA (Orbigen Inc.) into cultured Sf9 insect cells. The culture supernatants that contained recombinant viruses were infected into High Five cells, which were then cultured in serum-free EX-CELL 405 medium (JRH Biosciences Inc.) for 3 days. To express hDsg4-His in mammalian cells, pQE-hDsg4-His was transiently transfected into $\mathrm{CHO}$ cells using the Superfect Transfection Reagent (QIAGEN Inc.). To produce nonglycosylated forms of hDsg4-His, tunicamycin (Sigma-Aldrich) was added to culture media for High Five cells at $4.5 \mu \mathrm{g} / \mathrm{ml}$ and $\mathrm{CHO}$ cells at $0.5 \mu \mathrm{g} / \mathrm{ml}$ and incubated for 3 days.

$I P-I B$ analyses for detection of reactivity against desmogleins. To determine whether pemphigus sera contained IgG against Dsg4, a combined IP and IB analysis was performed using culture supernatants that contained either hDsg4-His or mDsg4-His as substrates. The culture supernatants were incubated with pemphigus sera or normal control sera and then immunoprecipitated with protein G-Sepharose or protein A-Sepharose (Amersham Biosciences) at $4{ }^{\circ} \mathrm{C}$ overnight with gentle rotation. The immunoprecipitants were then resuspended in SDS sample buffer $(62.5 \mathrm{mM}$ Tris-HCL, pH 7.5, 1\% SDS, $0.0025 \%$ bromophenol blue, $10 \%$ glycerol, $2.5 \%$ 2-mercaptoethanol), separated by SDS-PAGE, and transferred to a PVDF membrane (Millipore Corp.). The IP hDsg4-His and mDsg4-His proteins were visualized with the mouse anti-E tag mAb (Pharmacia Biotech).

To determine whether the recognition of Dsg4 by pemphigus sera was dependent on protein conformation, the culture supernatants that contained hDsg4-His were dialyzed against Tris-buffered saline in the absence of calcium and subjected to IP, as described above.

Immunoadsorption of pemphigus sera with Dsg1 and Dsg4. Sera were preincubated at $4^{\circ} \mathrm{C}$ for 1 hour with culture supernatant containing hDsg1-IgHis (25) or hDsg4-IgHis without the E-tag or with control culture supernatant without recombinant protein. Subsequently, the samples were incubated at $4{ }^{\circ} \mathrm{C}$ overnight with culture supernatants that contained hDsg1-His, hDsg3-His (33), or hDsg4-His, and immunoprecipitated with protein G-Sepharose. The IP hDsg1-His, hDsg3-His, and hDsg4-His proteins were detected specifically by IB analysis using the anti-E-tag $\mathrm{mAb}$.

Competition ELISA against Dsg1 using hDsg4-His as a competitor. To measure Dsg4-reactive IgG in anti-Dsg1 IgG autoantibodies in pemphigus, we performed competition ELISA against Dsg1 using hDsg4-His as a competitor. Pemphigus sera that reacted with Dsg4 by IP-IB analyses (11 PF sera and 12 mucocutaneous PV sera) were preincubated at $4{ }^{\circ} \mathrm{C}$ overnight with various amounts of hDsg4-His or hDsg1-His as a positive control for competition. The sera were then subjected to Dsg1 ELISA. When necessary, sera were diluted to keep the absorbance at $450 \mathrm{~nm}\left(\mathrm{~A}_{450}\right)$ below 1.2 in order to obtain a proper competition rate. Competition by immunoadsorption was calculated using the formula: competition $(\%)=\left(1-\left(\mathrm{A}_{450} \exp \right.\right.$ $\left.-\mathrm{A}_{450} \mathrm{pos}\right) /\left(\mathrm{A}_{450}\right.$ neg $\left.\left.-\mathrm{A}_{450} \mathrm{pos}\right)\right) \times 100 . \mathrm{A}_{450}$ pos and $\mathrm{A}_{450}$ neg are the measurements obtained for sera preincubated with a maximum dose of hDsg1-His and culture supernatant of uninfected High Five cells, respectively; $\mathrm{A}_{450} \exp$ is the measurement obtained for sera preincubated with various concentrations of hDsg4-His or hDsg1-His (31).

RT-PCR for detection of mouse Dsg expression. Total RNA was extracted from various sites of the skin (ventral skin, dorsal skin, footpad, tail), tongue, and liver of neonatal ICR mice ( $<24$ hours old) using RNeasy mini kit (QIAGEN Inc.). For footpad and tail samples the skin was carefully removed under a dissecting microscope. These RNA samples were used as templates to amplify mouse Dsg4, Dsg1 $\alpha$, Dsg1 $\beta$, Dsg1 $\gamma$, and Dsg3 by RT-PCR with Superscript One-Step RT-PCR (Invitrogen Corp.) and the following primers: mouse Dsg4 forward primer, 5'-CCTGGGTGACTGATGACATGTA-3'; mouse Dsg4 reverse primer, 5'-CTTGGTATTCCCGAAGAGTCCCTA-3'; mouse Dsg $1 \alpha$ and Dsg $1 \beta$ forward primer, $5^{\prime}$ - GGAACAATACCAAAGGCTTAATGG-3'; mouse Dsg1 $\alpha$ reverse primer, $5^{\prime}$ - GACGCGTTGTGGGTTCTCAGTGGAAGA-3'; mouse Dsg1 $\beta$ reverse primer, 5'-AGGCTCGAGGTGAACGTTGTCTTCTGTGATG-3'; mouse Dsg1 $1 \gamma$ forward primer, 5'-CAGGTCAAGCTACAAACAAGT-3'; mouse Dsg1 $\gamma$ reverse primer, 5'TGCCTCGAGGAAGTGAACATTGTCTACGTTTGGAGTG-3'; mouse Dsg3 forward primer, 5'-CCGGAATTCAGCATGTGCAGAGCCCCTATC3'; mouse Dsg3 reverse primer, 5'- GCTCTAGATCATTAGGTAATCATTTCCATTCCTGAAGCA-3'.

Passive transfer experiments with neonatal mice. Large-scale immunoadsorption of PF sera was performed using the AKTA explorer HPLC system (Amersham Biosciences) with the TALON affinity resin column (BD Biosciences - Clontech). Typically, 5-10 $\mathrm{ml}$ of PF sera was applied to the affinity resin column along with approximately 500-2,000 $\mu \mathrm{g} \mathrm{hDsg4-His} \mathrm{or}$ hDsg1-His, and IgG was precipitated from the flow-through fraction with $40 \%$ ammonium sulfate. The IgG fraction was then dialyzed against PBS and concentrated approximately tenfold using the microconcentrator Centriprep 30 (Amicon; Millipore Corp.). The complete removal of the desired IgG was confirmed by IP-IB analyses for anti-Dsg4 IgG and ELISA for antiDsg1 IgG. For affinity-purification, IgG bound to hDsg4-His or hDsg1-His was eluted with $0.1 \mathrm{M}$ glycine- $\mathrm{NaOH}$ ( $\mathrm{pH}$ 11.5), immediately neutralized with $1 \mathrm{M}$ Tris- $\mathrm{HCl}$ ( $\mathrm{pH} 7.4$ ), dialyzed against PBS, and concentrated to a final volume of $600 \mu \mathrm{l}$ in Centriprep 30 .

To evaluate the pathogenic activities of the immunoadsorbed sera, a passive transfer model with neonatal mice was used, as described previously $(26,43)$. Neonatal ICR mice at 12-24 hours of age (body weight, 1.6-1.8 g) were injected subcutaneously with the IgG fractions prepared as above. To compare the pathogenic strength of PF sera with and without Dsg4 immunoadsorption in a more quantitative fashion, the concentrated IgG fractions prepared by ammonium sulfate precipitation were serially diluted and injected into neonatal mice. Similarly, to compare the pathogenic activity of IgG affinity-purified on hDsg4-His or hDsg1-His, purified IgG was serially diluted and injected into neonatal mice. The mice were examined and a biopsy was performed when they developed blisters, or they were observed for 24 hours when they did not develop apparent blisters. All mouse studies were approved by the animal ethics review board of Keio University.

In vitro digestion of recombinant Dsg4 with ETs. Three isoforms of ET, ETA, ETB, and ETD, were prepared as described previously $(17,18)$. The culture supernatants that contained hDsg1-His, hDsg4-His, mDsg1 $\alpha$-His (40), or $\mathrm{mDsg} 4$-His were incubated with ETA, ETB, and ETD for 1 hour at $37^{\circ} \mathrm{C}$. The treated culture supernatants were subjected to IB analysis with the anti-E-tag $\mathrm{mAb}$ to visualize intact as well as digested recombinant proteins. 


\section{Acknowledgments}

We would like to thank Takeji Nishikawa for insightful discussion and review of this manuscript. We also thank Yoshiko Fujii and Minae Suzuki for technical assistance with recombinant protein preparation, cell culture, and immunofluorescence staining. This work was supported by Health and Labour Sciences Research Grants for Research on Measures for Intractable Diseases from the Ministry of Health, Labor, and Welfare and by Grants-in-Aid for Scientific Research from the Ministry of Education, Culture, Sports, Science, and Technology of Japan.

1. Green, K.J., and Gaudry, C.A. 2000. Are desmosomes more than tethers for intermediate filaments? Nat. Rev. Mol. Cell Biol. 1:208-216.

2. Runswick, S.K., O'Hare, M.J., Jones, L., Streuli, C.H., and Garrod, D.R. 2001. Desmosomal adhesion regulates epithelial morphogenesis and cell positioning. Nat. Cell Biol. 3:823-830.

3. Koch, P.J., et al. 1990. Identification of desmoglein, a constitutive desmosomal glycoprotein, as a member of the cadherin family of cell adhesion molecules. Eur. J. Cell Biol. 53:1-12.

4. Nilles, L.A., et al. 1991. Structural analysis and expression of human desmoglein: a cadherin-like component of the desmosome. J. Cell Sci. 99:809-821.

5. Amagai, M., Klaus-Kovtun, V., and Stanley, J.R 1991. Autoantibodies against a novel epithelia cadherin in pemphigus vulgaris, a disease of cell adhesion. Cell. 67:869-877.

6. Schafer, S., Koch, P.J., and Franke, W.W. 1994. Identification of the ubiquitous human desmoglein, Dsg2, and the expression catalogue of the desmoglein subfamily of desmosomal cadherins. Exp. Cell Res. 211:391-399.

7. Kljuic, A., et al. 2003. Desmoglein 4 in hair follicle differentiation and epidermal adhesion: evidence from inherited hypotrichosis and acquired pemphigus vulgaris. Cell. 113:249-260.

8. Whittock, N.V., and Bower, C. 2003. Genetic evidence for a novel human desmosomal cadherin, desmoglein 4. J. Invest. Dermatol. 120:523-530.

9. Rickman, L., et al. 1999. N-terminal deletion in a desmosomal cadherin causes the autosomal dominant skin disease striate palmoplantar keratoderma. Hum. Mol. Genet. 8:971-976.

10. Amagai, M. 2003. Pemphigus. In Dermatology. J. Bolognia et al., editors. Harcourt Health Sciences. London, United Kingdom. 449-462.

11. Ding, X., et al. 1997. Mucosal and mucocutaneous (generalized) pemphigus vulgaris show distinct autoantibody profiles. J. Invest. Dermatol. 109:592-596.

12. Amagai, M., Tsunoda, K., Zillikens, D., Nagai, T., and Nishikawa, T. 1999. The clinical phenotype of pemphigus is defined by the anti-desmoglein autoantibody profile. J. Am. Acad. Dermatol. 40:167-170.

13. Mahoney, M.G., et al. 1999. Explanation for the clinical and microscopic localization of lesions in pemphigus foliaceus and vulgaris. J. Clin. Invest. 103:461-468

14. Amagai, M. 1999. Autoimmunity against desmosomal cadherins in pemphigus. J. Dermatol. Sci. 20:92-102

15. Ladhani, S., Joannou, C.L., Lochrie, D.P., Evans, R.W., and Poston, S.M. 1999. Clinical, microbial, and biochemical aspects of the exfoliative toxins causing staphylococcal scalded-skin syndrome.

Received for publication November 6, 2003, and accepted in revised form September 21, 2004.

Address correspondence to: Masayuki Amagai, Department of Dermatology, Keio University School of Medicine, 35 Shinanomachi, Shinjuku-ku, Tokyo 160-8582, Japan. Phone: 81-3-5363-3425; Fax: 81-3-3351-6880; E-mail: amagai@sc.itc.keio.ac.jp.

Takeshi Nagasaka and Koji Nishifuji contributed equally to this work.

Clin. Microbiol. Rev. 12:224-242.

16. Amagai, M., Matsuyoshi, N., Wang, Z.H., Andl, C., and Stanley, J.R. 2000. Toxin in bullous impetigo and staphylococcal scalded skin syndrome targets desmoglein 1. Nat. Med. 6:1275-1277.

17. Amagai, M., et al. 2002. Staphylococcal exfoliative toxin B specifically cleaves desmoglein 1 . J. Invest. Dermatol. 118:845-850.

18. Yamaguchi, T., et al. 2002. Identification of Staphylococcus aureus etd pathogenicity island which encodes a novel exfoliative toxin, ETD, and EDIN-B Infect. Immun. 70:5835-5845.

19. Hanakawa, Y., et al. 2002. Molecular mechanisms of blister formation in bullous impegito and staphylococcal scalded skin syndrome. J. Clin. Invest. 110:53-60. doi:10.1172/JCI200215766.

20. Rafiq, M.A., et al. 2004. A recurrent intragenic deletion mutation in DSG4 gene in three Pakistani families with autosomal recessive hypotrichosis. J. Invest. Dermatol. 123:247-248.

21. Moss, C., et al. 2004. A recurrent intragenic deletion in the desmoglein 4 gene underlies localized autosomal recessive hypotrichosis. J. Invest. Dermatol. 123:607-610.

22. Jahoda, C.A., et al. 2004. The lanceolate hair rat phenotype results from a missense mutation in a calcium coordinating site of the desmoglein 4 gene. Genomics. 83:747-756.

23. Sundberg, J.P., et al. 2000. Lanceolate hair-J (lahJ): a mouse model for human hair disorders. Exp. Dermatol. 9:206-218.

24. Harman, K., Gratian, M., Bhogal, B., Challacombe, S., and Black, M. 2000. A study of desmoglein 1 autoantibodies in pemphigus vulgaris: racial differences in frequency and the association with a more severe phenotype. Br. J. Dermatol. 143:343-348.

25. Amagai, M., Nishikawa, T., Nousari, H.C., Anhalt, G.J., and Hashimoto, T. 1998. Antibodies against desmoglein 3 (pemphigus vulgaris antigen) are present in sera from patients with paraneoplastic pemphigus and cause acantholysis in vivo in neonatal mice. J. Clin. Invest. 102:775-782.

26. Anhalt, G.J., Labib, R.S., Voorhees, J.J., Beals, T.F., and Diaz, L.A. 1982. Induction of pemphigus in neonatal mice by passive transfer of IgG from patients with the disease. N. Engl. J. Med. 306:1189-1196.

27. Pulkkinen, L., Choi, Y.W., Kljuic, A., Uitto, J., and Mahoney, M.G. 2003. Novel member of the mouse desmoglein gene family: Dsg1- $\beta$. Exp. Dermatol. 12:11-19.

28. Kljuic, A., and Christiano, A.M. 2003. A novel mouse desmosomal cadherin family member, desmoglein 1 $\gamma$. Exp. Dermatol. 12:20-29.

29. Stanley, J.R. 1993. Cell adhesion molecules as targets of autoantibodies in pemphigus and pemphigoid, bullous diseases due to defective epidermal cell adhesion. Adv. Immunol. 53:291-325.

30. Futei, Y., et al. 2000. Conformational eptiope mapping of desmoglein 3 using domain-swapped molecules in pemphigus vulgaris. J. Invest. Dermatol. 115:829-834

31. Sekiguchi, M., et al. 2001. Dominant autoimmune epitopes recognized by pemphigus antibodies map to the $\mathrm{N}$-terminal adhesive region of desmogleins. J. Immunol. 167:5439-5448.

32. Boggon, T.J., et al. 2002. C-cadherin ectodomain structure and implications for cell adhesion mechanisms. Science. 296:1308-1313.

33. Ishii, K., et al. 1997. Characterization of autoantibodies in pemphigus using antigen-specific ELISAs with baculovirus expressed recombinant desmogleins. J. Immunol. 159:2010-2017.

34. Ota, T., Amagai, M., Watanabe, M., and Nishikawa, T. 2003. No involvement of IgG autoantibodies against extracellular domains of desmoglein 2 in paraneoplastic pemphigus or inflammatory bowel diseases. J. Dermatol. Sci. 32:137-141.

35. Schafer, S., Stumpp, S., and Franke, W.W. 1996. Immunological identification and characterization of the desmosomal cadherin Dsg2 in coupled and uncoupled epithelial cells and in human tissues. Differentiation. 60:99-108.

36. Kurzen, H., et al. 1998. Compositionally different desmosomes in the various compartments of the human hair follicle. Differentiation. 63:295-304.

37. Wu, H., Stanley, J.R., and Cotsarelis, G. 2003. Desmoglein isotype expression in the hair follicle and its cysts correlates with type of keratinization and degree of differentiation. J. Invest. Dermatol. 120:1052-1057.

38. Koch, P.J., et al. 1997. Targeted disruption of the pemphigus vulgaris antigen (desmoglein 3 ) gene in mice causes loss of keratinocyte cell adhesion with a phenotype similar to pemphigus vulgaris. J. Cell Biol. 137:1091-1102.

39. Koch, P.J., et al. 1998. Desmoglein 3 anchors telogen hair in the follicle. J. Cell. Sci. 111:2529-2537.

40. Amagai, M., et al. 2000. Use of autoantigen knockout mice to develop an active autoimmune disease model of pemphigus. J. Clin. Invest. 105:625-631.

41. Amagai, M., et al. 1999. Usefulness of enzymelinked immunosorbent assay (ELISA) using recombinant desmogleins 1 and 3 for serodiagnosis of pemphigus. Br. J. Dermatol. 140:351-357.

42. Tsunoda, K., et al. 2003. Induction of pemphigus phenotype by a mouse monoclonal antibody against the amino-terminal adhesive interface of desmoglein 3. J. Immunol. 170:2170-2178.

43. Amagai, M., Hashimoto, T., Shimizu, N., and Nishikawa, T. 1994. Absorption of pathogenic autoantibodies by the extracellular domain of pemphigus vulgaris antigen (Dsg3) produced by baculovirus. J. Clin. Invest. 94:59-67. 